\section{Recurrent supraventricular tachycardia and necrotizing enterocolitis: A causative role or a simple association? A case report and literature review}

\author{
Ghassan Nakib, ${ }^{1}$ Sameer Sajwani, ${ }^{2}$ \\ Zahreddin Abusalah, ${ }^{3}$
}

Abdelazeim Abdallah, ${ }^{3}$ Nada Ibrahim, ${ }^{4}$

Aseel Fattah, ${ }^{4}$ Rossana Bussani, 5

Valeria Calcaterra, ${ }^{6}$ Gloria Pelizzo ${ }^{7}$

1Department of Paediatric Surgery, Medclinic Middle East, Mediclinic City

Hospital Dubai, UAE; 2Department of Paediatric, Pediatric Cardiology Unit, Mediclinic City Hospital, Dubai, UAE; 3Department of Neonatology, Mediclinic City Hospital, Dubai, UAE;

${ }^{4}$ Histopathology Unit, Mediclinic City

Hospital, Dubai, UAE; 5Institute of

Pathologic Anatomy, University of

Trieste, Italy; ${ }^{\circ}$ Pediatric Unit,

Department of Maternal and Children's

Health, Fondazione IRCCS Policlinico

San Matteo and Department of Internal

Medicine, University of Pavia, Pavia,

Italy; ${ }^{7}$ Department of Pediatric Surgery,

Children's Hospital "G. Di Cristina,

ARNAS Civico-Di Cristina-Benfretelli”,

Palermo, Italy

\begin{abstract}
Insufficiency in mesenteric flow is a risk factor for the development of necrotizing enterocolitis (NEC). Recurrent episodes of supraventricular tachycardia (SVT) can lead to gut ischemia, with subsequent reperfusion injury. We present a term infant who developed NEC at 37 days of life after refractory SVT and reverted to sinus rhythm on day 23 and 25 of life. Resected stenotic ileum and transverse colon demonstrated inflammation with ischemia. This is the first case of NEC following SVT in a term infant without a congenital morphologic abnormality. In view of the temporal sequence of events, and in the absence of other risk factors for NEC, the SVT and NEC were likely causally related.
\end{abstract}

\section{Introduction}

Necrotizing enterocolitis (NEC) is a common and devastating multifactorial condition in neonates. ${ }^{1-6}$ The pathogenesis of NEC is incompletely understood, but epi- demiological observations strongly suggest a multifactorial cause. 4-6 In full-term newborns several risk factors contribute to NEC, including postnatal asphyxia, meconium aspiration syndrome, sepsis, congenital heart disease (CHD). ${ }^{7}$ The finding that the risk for NEC is correlated with asphyxia also strongly supports the notion that insufficiency in mesenteric blood flow is a risk factor for NEC development. ${ }^{8}$

Supraventricular tachycardia (SVT) is the most common tachyarrhythmia in children. Recurrent episodes of the SVT can lead to gut ischemia, with subsequent reperfusion injury. ${ }^{9}$

The association between NEC and SVT had been previously described in pre-term babies, or term infants with CHD or older than one year. In order to help frame the contribution to the literature, we present the case of a full-term infant with recurrent SVT, without CHD, who developed NEC in the first months of life. Intestinal ischemia due to SVT could have been a precursor leading to NEC in this patient.

\section{Case Report}

A 37-day-old girl was admitted to our Pediatric Surgery Unit with abdominal obstruction.

The patient was born at 38 weeks' gestation (birth weight $2755 \mathrm{~g}$ ). She was breastfed at birth. On day 13, the physical evaluation in the primary pediatric care setting revealed tachycardia with a heart rate (HR) of more than 200 beats per minute (bpm). No vomiting, constipation or abdominal distension were noted. The asymptomatic girl was admitted to the Neonatal Intensive Care Unit for further investigation.

On admission, the 13 days old term infant had a SVT of 278-294 bpm which was refractory to vagal stimulation, electrical cardioversion, and repeated doses of adenosine. She was reverted to sinus rhythm with amiodarone and flecainide on the 4th day of admission. Echocardiography showed normal heart anatomy, borderline and dyskinetic cardiac function and a left ventricle ejection fraction of $50 \%$. Day 23 of life she again developed SVT, and for the 3 rd time on day 25 , both episodes reverted to sinus rhythm by adenosine. In conclusion she had refractory SVT initially for 4 days followed by 2 episodes of recurrent SVT, which were not refractory and responded to adenosine.

Abdominal distension was initially noted on day 16 (3 days after admission) while still in the phase of refractory SVT, for which she received glycerin suppository,
Correspondence: Ghassan Nakib, Department of Pediatric Surgery, Medclinic Middle East, Mediclinic City Hospital Dubai, UAE.

Tel.: +971569820815.

E-mail: Ghassan.Nakib@mediclinic.ae

Key words: Necrotizing enterocolitis; supraventricular tachycardia; recurrent; newborn; term.

Acknowledgements: the authors would thank Prof. C.V. Bellieni for your expertise.

Contributions: GN surgical management of the patient, drafting the article, critical revision of the article; SS cardiological managment of the patient, drafting the article; ZA neonatal management of the patient, drafting the article; NA, $\mathrm{AF}, \mathrm{RB}$, histological analysis, drafting the article; VC, drafting the article, literature review, critical revision of the article; GP, drafting the article, literature review, critical revision of the article

Conflict of interests: the authors declare that they have no conflict of interests.

Received for publication: 10 February 2018.

Revision received: 11 June 2018.

Accepted for publication: 11 June 2018.

This work is licensed under a Creative Commons Attribution NonCommercial 4.0 License (CC BY-NC 4.0).

(C) Copyright G. Nakib et al., 2018

Licensee PAGEPress, Italy

Pediatric Reports 2018; 10:7636

doi:10.4081/pr.2018.7636

this was followed by decreased activity which was attributed to sepsis and treated with antibiotics. The infection markers remained static and did not drop as expected in spite of 14 days on antibiotics. On day 32 of life, surgical evaluation was undertaken due to worsening abdominal distension. Intestinal obstruction was strongly suspected. Imaging showed dilated loops of bowel with no free air in the abdomen. On contrast enema severe stenosis at the transverse colon, with dilated small bowel loops, were seen (Figure 1). During preparation for laparotomy, she developed fecal vomiting (sign of distal small bowel or colonic obstruction). At laparotomy the terminal ileum and a jejunal loop with omentum were solidly adherent to the stenotic transverse colon; a sign of sealed perforation. Resection of the stenotic transverse colon was done with end-end anastomosis, and ileal resection of a stenotic segment $(2 \mathrm{~cm})$ with end-end anastomosis. Histologically, there was ulceration, transmural inflammation and granulation tissue in the colon (Figure 2, Panels A-C). She was restarted 
on enteral feeds on day 5 post surgery and was gradually built up to full feeds.

Following surgery, she continued on oral amiodarone. On day 24 her cardiac ultrasound reported normal findings with good function. Postoperative progress was uneventful and the patient was discharged home on the $7^{\text {th }}$ postoperative day.

She has regular follow up, and is on oral amiodarone which is planned to continue till the age of 1 year. The parents have elected to have continuous home monitoring of her heart rate. She had Holter monitoring done twice and no rhythm alteration were detected on both occasion.

\section{Discussion and Conclusions}

NEC remains a major cause of morbidity and mortality in infants. ${ }^{4,5}$ Commonly observed risk factors for NEC are prematurity, low birth-weight, postnatal asphyxia, sepsis, enteral feeding, hypothermia, blood transfusion, meconium aspiration syndrome and CHD. It is widely believed that intestinal ischaemia is the unifying mechanism that explains the ability of these many different factors to cause injury to the neonatal bowel. Risk factors might cause ischaemia directly or as a consequence of the 'diving

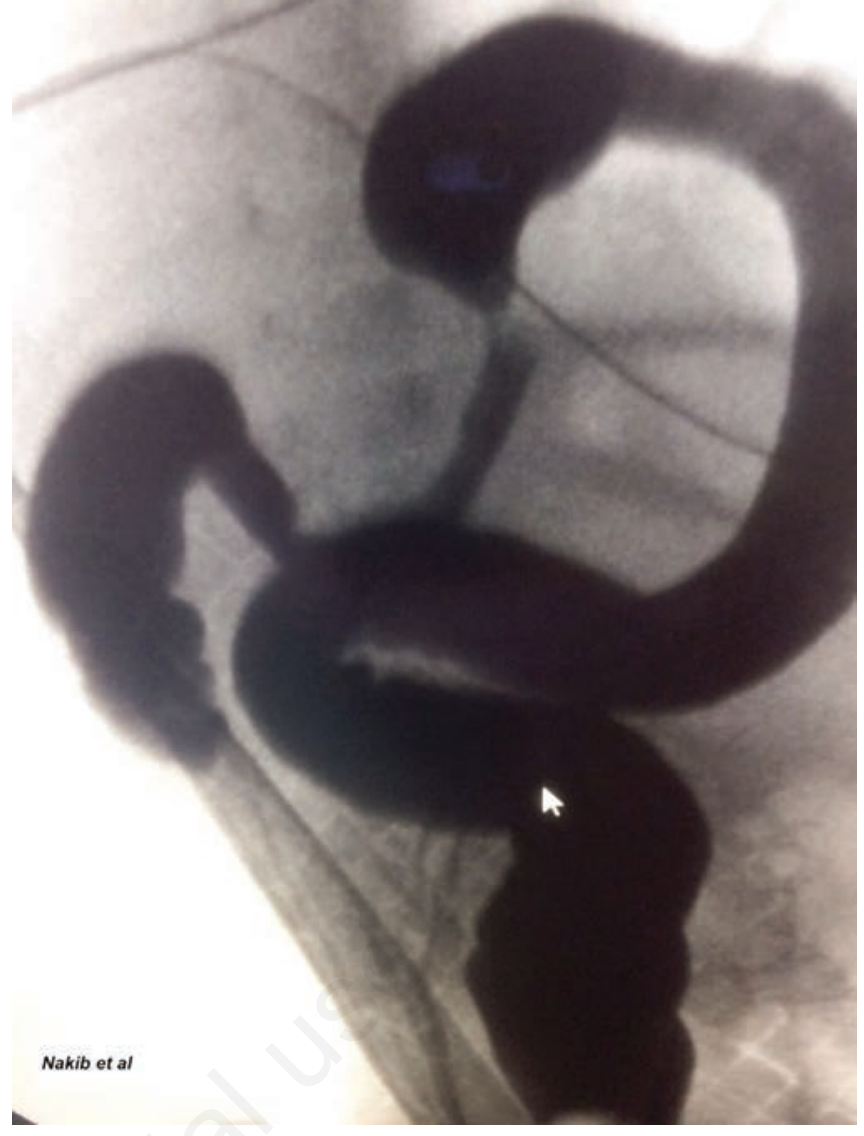

Figure 1. Contrast enema: stenosis of transverse colon.

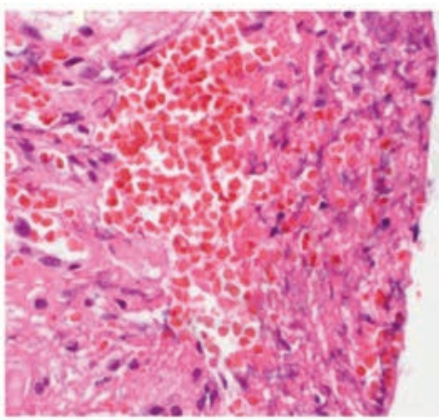

A

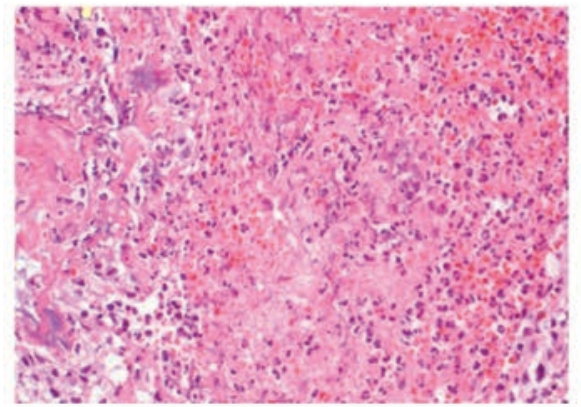

C

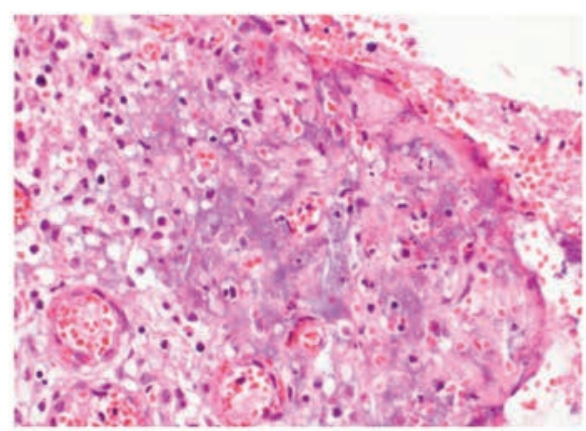

B

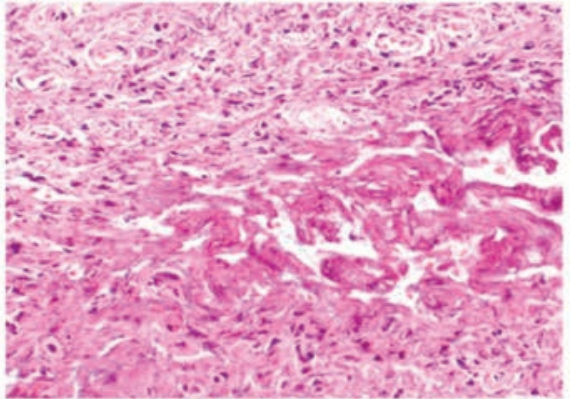

$\mathrm{D}$

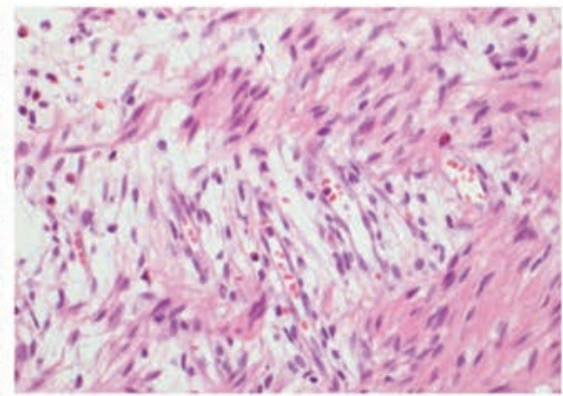

$\mathrm{E}$

Figure 2. Histology in the resected intestinal segments at the side of stenosis (hematoxylin and eosin stain). A) colon, presence of granulation tissue and smudget nuclei at lumen with ischemic changes $(60 \mathrm{X}) ; \mathrm{B})$ colon, sloughand granulation tissue at luminal aspect (40X); C) colon, slough purulent (40X); D) colon, slough at sierosa (40X); E) colon, disrupted muscle edema and congestion (40X). 
reflex' when blood flow is diverted from the abdominal organs during hypoxia, thus preserving cerebral oxygenation. 7,8

We presented a case of NEC in an infant with refractory SVT despite combined medical treatment. There are six previous case reports in the literature of NEC following neonatal SVT; this is a first case in a term infant, breastfed at birth, without congenital cardiac disease that has developed NEC in the first months of life. Saini et al. ${ }^{10}$ presented two cases of late preterm infants with NEC, characterised by pneumatosis on abdominal X-ray and ultrasound, following recurrent SVT without haemodynamic instability. Hanna ${ }^{11}$ described a preterm infant with recurrent episodes of SVT who developed NEC in the second week of life. Jacombs et al.12 reported a 15-month-old boy who presented in shock with SVT a following a 12-hour history of worsening abdominal pain and vomiting, in which a diagnosis of intestinal ischaemia was confirmed. Hajivassiliou et al., ${ }^{9}$ described a 5month-old baby with CHD and recurrent episodes of refractory SVT that developed severe segmental NEC after one prolonged episode. Khalak et al. ${ }^{13}$ reported a premature neonate with SVT that was treated prenatally and postnatally, without significant signs of congestive heart failure and that developed fatal, fulminant necrotizing enterocolitis 28 hours after starting feeds.

SVT is the most frequent form of symptomatic tachydysrhythmia in pediatric age. Atrioventricular nodal reentrant tachycardia represents the most common tachycardia substrate in infants and young children. ${ }^{14,15}$

The presentation of patients with SVT during the first year of life is highly variable. Infants are often asymptomatic and, as in our patient, SVT is diagnosed when tachycardia is observed by medical care providers or other caregivers. If the arrhythmia persists, the infants may develop nonspecific symptoms such as poor feeding, tachypnea, lethargy or pallor.

In the majority of infants with SVT, tachycardia is well tolerated and should be managed conservatively. If SVT does not resolve spontaneously and symptoms are present, cardioversion using vagal maneuvers, therapy with IV adenosine or IV esmolol, transesophageal pacing or synchronized DC current should be attempted. If SVT recurs, or if symptoms are severe, then chronic medication treatment may be warranted. 14

Persistent tachycardias can lead to hemodynamic compromise ${ }^{16}$ and represent a potential risk of gut hypoperfusion and ischemia because of a combination of high systemic and splanchnic venous pressures (persistent tachycardia, low cardiac output).$^{9}$

Ischemia appears to be an important contributing factor to the development of necrotizing enterocolitis. In particular, reperfusion plays a major role in ischemiarelated (I/R) injury, and intestinal mucosal permeability and oxygen free radicals produced during reperfusion most likely contribute to the injury. Many observations that specific tissues/organs, such as neuronal and gastro-intestinal tissue, are more susceptible to $\mathrm{I} / \mathrm{R}$ injury exist. ${ }^{17-19}$

In our patient, the abdominal signs, along with signs of sepsis, started after 3 days of refractory SVT. In view of the temporal sequence of events, in the absence of the other major etiologic risk factors for NEC, it is more likely that the SVT and NEC were causally related. Decreased visceral blood flow is not recognised as a side effect of any of the antiarrhythmic agents used in our case, however their potential role on systemic blood pressure and cardiac ejection may have contribute to impaired blood flow. This baby may have had a period of compromise of his visceral circulation secondary to her SVT with subsequent reperfusion injury. At presentation with SVT, the baby was asymptomatic with no suspicion of abdominal pathology. Although the symptoms of acute intestinal ischemia can be variable, in our case, classical abdominal signs and positive indicators of underlying sepsis (high CRP and decreased motor activity) some days after admission, with negative baseline indicators, further supports our conclusion. The sequence of events almost excludes the idea of acute intestinal ischaemia preceding and triggering the SVT.

In conclusion, our report and literature review suggest that SVT, which can led to intestinal ischemia, was probably the main causative factor for this patient's NEC. ${ }^{9}$

\section{References}

1. Llanos AR, Moss ME, Pinzo'n MC, et al. Epidemiology of neonatal necrotising enterocolitis: a population-based study. Paediatr Perinat Epidemiol 2002;16: 342-9.

2. Andrews DA, Sawin RS, Ledbetter DJ, et al. Necrotizing enterocolitis in term neonates. Am J Surg 1990;159:507-9.

3. Ostlie DJ, Spilde TL, St Peter SD, et al. Necrotizing enterocolitis in full-term infants. J Pediatr Surg 2003;38:103942.

4. Neu J, Walker WA. Necrotizing enterocolitis. N Engl J Med 2011;364:255-64.
5. Horton KK. Pathophysiology and current management of necrotizing enterocolitis. Neonatal Netw 2005;24:37-46.

6. Frost BL, Modi BP, Jaksic T, et al. New medical and surgical insights into neonatal necrotizing enterocolitis:a review. JAMA Pediatr 2017;171:83-8.

7. Lu Q, Cheng S, Zhou M, et al. Risk Factors for necrotizing enterocolitis in neonates: a retrospective case-control study. Pediatr Neonatol 2017;58:16570.

8. Kempley ST, Gamsu HR. Superior mesenteric artery blood flow velocity in necrotising enterocolitis. Arch Dis Child 1992;67:793-6.

9. Hajivassiliou CA, Pitkin J. Recurrent necrotizing enterocolitis associated with episodes of supraventricular tachycardia. J Pediatr Surg 1998;33:1569-70.

10. Saini J, Moore A, Hodgson K. Necrotising enterocolitis after supraventricular tachycardia: an unusual precursor to a common problem. BMJ Case Rep 2017;16;2017.

11. Hanna MH. Necrotizing enterocolitis after recurrent supraventricular tachycardia. J Neonatal Perinatal Med 2013; 6:359-61

12. Jacombs AS, Cross KM, Holland AJ, et al. Supraventricular tachycardia as a presenting feature of volvulus in a 15month-old boy. J Paediatr Child Health 2009;45:389-90.

13. Khalak R, Chess PR. Fulminant necrotizing enterocolitis in a premature neonate treated for supraventricular tachycardia. J Perinatol 1998;18:306-7.

14. Etheridge SP, Judd VE. Supraventricular tachycardia in infancy: evaluation, management, and follow-up. Arch Pediatr Adolesc Med 1999;153:267-71.

15. Etheridge SP, Saarel EV. The infant with supraventricular tachycardia: diagnosis and management. Progress Pediatr Cardiol 2013;35:1-6.

16. Li X, Zhang Y, Liu H, et al. Efficacy of intravenous sotalol for treatment of incessant tachyarrhythmias in children. Am J Cardiol 2017;119:1366-70.

17. Abela CB, Homer-Vanniasinkham S. Clinical implications of ischaemiareperfusion injury. Pathophysiology 2003;9:229-40.

18. Choi DW. The role of glutamate neurotoxicity in hypoxic-ischaemic neuronal death, Annu Rev Neurosci 1190;13:171.

19. Carati CJ. Changes in macromolecular permeability of microvessels in rat small intestine after total occlusion ischaemia/reperfusion. Microcirc Endothel Lymph 1988;5:157-70. 